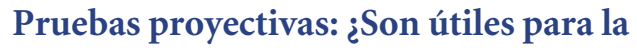 evaluación infantil?}

Projective tests: Are they useful for child assessment?

\section{Sr. Editor}

Las Pruebas Proyectivas (PP) son técnicas que utilizan los profesionales de la salud mental para evaluar aspectos emocionales y de personalidad, a través de la presentación de estímulos ambiguos, que deben ser respondidos por el evaluado ${ }^{1}$. Estas técnicas de evaluación tienen ciertos cuestionamientos acerca de su validez para detectar y discriminar a niños con trastornos emocionales, conductuales, cognitivos ${ }^{2}$, abuso sexual y/o físico ${ }^{3,4}$, principalmente porque estas pruebas tienen una gran cantidad de indicadores, situación que dificulta su replicación ${ }^{3,4}$.

En defensa de las PP, algunos autores argumentan que estas son solo parte de un conjunto más amplio de instrumentos, debiendo tomarse más pruebas para verificar lo encontrado. Pese a ello, este argumento ostenta ciertas críticas, puesto que un aumento en la cantidad de instrumentos, en vez de verificar lo obtenido, podría contaminar la información dificultando el juicio clínico ${ }^{3,5}$. En otras palabras, es como ir al médico y solicitar tres exámenes de biopsia cuando en realidad con uno es suficiente para el diagnóstico de cáncer.

La suposición de que un dibujo refleja en forma verídica características psicológicas del infante es cuestionable si no se consideran factores asociados como la experiencia previa, intereses personales, creatividad, problemas motrices, entre otros ${ }^{6}$. Por lo tanto, su utilización e interpretación debe darse a la luz de la historia clínica, en cuyo caso ha demostrado utilidad para detectar niños angustiados (al menos cuando se utilizan los mismos indicadores $)^{4} y$ niños con retraso en su desarrollo motriz y cognitivo ${ }^{6}$. A pesar de eso, en algunos contextos las PP se siguen utilizando de forma indiscri- minada, produciendo falsos positivos en la detección y diagnóstico de desórdenes conductuales, cognitivos o del aprendizaje ${ }^{2}$, lo que en nuestra opinión podría determinar una acción iatrogénica.

En conclusión, las PP son técnicas poco efectivas para la detección de desórdenes emocionales, conductuales, cognitivos ${ }^{2}$, abuso sexual y/o físico ${ }^{3,4}$, pero es importante recalcar que podrían utilizarse como instrumentos de detección preliminar siempre y cuando estén a la luz de una evaluación clínica profunda del paciente. De ese modo, es recomendable no basar el diagnóstico exclusivamente en estos instrumentos, pues existe un conjunto de técnicas con mayor evidencia científica (como la entrevista clínica, la observación conductual o pruebas psicométricas) para obtener un diagnóstico preciso y confiable en la evaluación infantil.

\section{Andy Rick Sánchez-Villena ${ }^{a}$, José Ventura-León ${ }^{a}$ ${ }^{a}$ Universidad Privada del Norte, Perú.}

\section{Referencias}

1. Hunsley J, Lee K, Wood J, Taylor W. Controversial and Questionable assessment techniques. In: Lilienfeld S, Lynn S, Lohr J, editors. Science and Pseudoscience in Clinical Psychology. 2nd ed. New York: The Guildford Press; 2015. p. 42-83.

2. Chollat C, Joly A, Houivet E, Bénichou J, Marret S. School-age human figure drawings by very preterm infants: Validity of the Draw-a-Man test to detect behavioral and cognitive disorders. Arch. Pédiatrie. 2019; 26(4):220-5.

3. Allen B, Tussey C. Can Projective Drawings Detect if a Child Experienced Sexual or Physical Abuse? Trauma Violence Abuse. 2012; 13(2):97-111.

4. Garb HN, Wood JM, Nezworski MT. Projective Techniques and the Detection of Child Sexual Abuse. Child Maltreatment. 2000; 5(2):161-8.

5. Erickson SK, Lilienfeld SO, Vitacco MJ. A Critical Examination Of The Suitability And Limitations Of Psychological Tests In Family Court. Fam. Court Rev. 2007; 45(2):157-74.

6. Tükel Ş, Eliasson AC, Böhm B, Smedler AC. Simple Categorization of Human Figure Drawings at 5 Years of Age as an Indicator of Developmental Delay. Dev. Neurorehabil. 2019; 22(7):479-86.

Correspondencia: 